\title{
Power Gain Analysis of Optically Illuminated MOSFET
}

\author{
Prerana Jain \\ Ph D student \\ SKVM's NMIMS, Vile Parle(W) \\ Mumbai, India
}

\author{
B. K. Mishra \\ Principal \\ TCET, Kandivali (E) \\ Mumbai, India
}

\author{
Phade G. \\ Ph D student \\ SNDT University, Juhu \\ Mumbai, India
}

\begin{abstract}
Modelling of optically illuminated MOSFET is done considering the substrate effect to evaluate admittance and scattering parameters for microwave frequency applications. Analysis of various gains and figure of merit is also incorporated indicating that optically controlled MOSFET can be used in optical communication circuits with enhanced electrical characteristics.
\end{abstract}

\section{General Terms}

Modeling, Optoelectronics, Simulation.

\section{Keywords}

Modeling, MOSFET, Photodetector, optoelectronics, Powergain, RF

\section{INTRODUCTION}

CMOS is the best sub-micron technology and it is important economically also. [1] Due to several advantages of Silicon, there is impressive progress in this technology and has made the MOSFETs to operate at higher frequencies and achieve better operating gains. The performance of transistors at microwave is largely controlled by device characteristics. For the MOSFET, reduction in gate length, increase in device width and active layer characteristics play a major role [2]. For a feasible technology, a cost effective solution is desired. Optoelectronic and RF CMOS integration offers all the benefits like immunity to electromagnetic interference, better reliability etc [3] .Optically controlled MOSFET offers additional advantage of high gain photo detector and availability of an additional independent port to control the device characteristics and operating region which will be added advantage at RF. This will always improve the commercial viability of direct integration between monolithic microwave integrated circuits (MMICs), and Optoelectronic Integrated Circuits (OEICs).

The I-V characteristics are important at DC while Scattering (S) parameters and admittance $(\mathrm{Y})$ parameters are important at high frequency. Admittance or impedance parameters require short circuit or open circuit condition for measurement, which is difficult to achieve at high frequency where lead inductance and parasitic capacitances dominate the measurement. Sparameters are small signal parameters by definition and high frequency behaviour of the device is determined around a bias point over its operational bandwidth. The $\mathrm{S}$ parameters are also important as they are used to determine signal power gain and various figures of merit and also been discussed in detail in $[4,5]$.

A number of small signal models for MOSFETs at high frequency are proposed [6-8] .Equivalent circuit models are simple to use since they have advantage of easy analysis and have the potential to operate over wide range of conditions [9]. Y and S parameter analysis and simulation for the optically controlled MOSFET has already been done in [1012 . and it has been shown that real value of parameter y 22 in dark as well in illuminated condition does not match measured results. This is due to neglecting of substrate coupling at high frequency which has been discussed in [13]

This paper presents a simple and accurate model for small signal MOSFET including substrate related parameters and transcapacitances under dark and optical illumination .Y parameters are simulated using analytical expressions taking substrate induced effects into account. The $\mathrm{S}$ parameters are obtained from inter-conversion of $\mathrm{Y}$ parameters. Gain and feedback considerations are of prime importance while dealing with active devices and hence power gain issues and their graphical displays are the starting point of analysis and design of high frequency amplifiers. To meet this requirement the transducer gain, available power gain, operating power gain, unilateral power gain and maximum stable power gain are evaluated for dark and optical radiation. Analysis for figure of merit is also presented.

Section -II presents MOSFET under illumination and related analysis. Section III presents the small signal model for MOSFET at RF with substrate effects and the expressions for parameters, calculations of various gains and unilateral figure of merit.

\section{MOSFET UNDER ILLUMINATION}

The structure under consideration is an optically illuminated $\mathrm{N}$ - MOSFET as shown in Figure-1.MOSFETs at RF are often multifinger devices to reduce gate resistance $R g$. The figure represents a single finger operated at RF for analysis which has been in [14]. Optical radiation is perpendicular to the surface and the wavelength of radiation is higher than that of silicon band-gap energy and is considered to be of $830 \mathrm{~nm}$ for simulation purpose. Optical radiation is assumed to be incident in the $\mathrm{Y}$ direction.

Investigations have been done on an MIS device which can be modified to optically gated MISFET (OG-MISFET). One of the key device structures is $\mathrm{Si}-\mathrm{SiO} 2$, and the device can be referred as optically gated MOSFET (OG-MOSFET)[15]. The device is modelled at higher range of frequency where consideration to extrinsic components has to be given, as against in low and medium frequency range.

In N-MOSFET, the ion implanted channel profile in the active region of the device is represented by

$N(y)=\frac{Q}{\sigma \sqrt{2 \pi}} \exp \left(-\left(\frac{y-R p}{\sigma \sqrt{2}}\right)^{2}\right)$ 


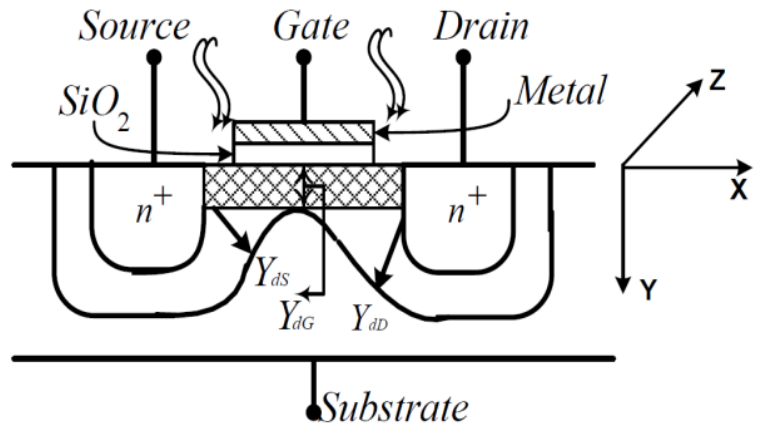

Figure 1 Schematic of MOSFET under optical radiation

Where $\mathrm{Q}$ is implanted dose, $\sigma$ straggle parameter, Rp projected range.

Illumination with source of wavelength less than critical wavelength causes the two effects. The incident radiation with source energy greater than bandgap of Silicon, results in generation of excess carriers. This increases the total channel charge resulting in enhancement of channel conductivity. Another effect of illumination is increase in channel dimensions due to photovoltaic effect across channel gate junction [16].

The total charge in channel in case of optical illumination is given by

$Q_{\text {total }}=Q_{\text {ion }}+Q_{\text {illumination }}$

$Q_{i o n}$ is charge due to ion implantation,

$\mathrm{Q}_{\text {illumination }}$ is induced charge due to illumination.

The charge due to illumination is due generated carriers in depletion and neutral region of the channel which contributes to total channel charge. The $Q_{i o n}$ and $\mathrm{Q}_{\text {illumination }}$ are calculated as per with appropriate boundary conditions [17 ].

The total drain source current under optical radiation is obtained as

$I_{d s}=\frac{\mu \mathrm{Z}}{\mathrm{L}} \int_{0}^{V_{D S}} Q_{\text {total }} d V$

The drain-source current flows along the $\mathrm{X}$ direction and the device is illuminated along the $\mathrm{Y}$ direction. The gate being opaque, the excess carriers are generated in the extended gate depletion region and the neutral region of the channel .The optically generated electrons flow toward the drain and contributes to the drain-source current when a drain source voltage is applied.

The external photovoltage developed across the junction is obtained using the relation as in[18]

$V o p=\frac{K T}{q} \ln \left(\frac{J p}{J_{S}}\right)=\frac{K T}{q} \ln \left(\frac{q v_{y} p(0)}{J_{S_{1}}}\right)$

Where Js is the reverse saturation current,

$\mathrm{v}_{\mathrm{y}}$ is the carrier along vertical direction perpendicular to the device surface,

$\mathrm{p}(0)$ is number of holes crossing junction at $\mathrm{y}=0$

$$
p(0)=\frac{\Pi}{4} Z\left(p_{1} Y d S^{2}+p_{2} Y d D^{2}\right)
$$

Where $\mathrm{p}_{1}$ and $\mathrm{p}_{2}$ are the constants dependant on carrier lifetime under ac conditions.

$\mathrm{Y}_{\mathrm{dD}}$ and $\mathrm{Y}_{\mathrm{dS}}$ is depletion width at drain and source resp.
The calculation of photovoltage is important as it modifies the depletion width $\mathrm{Y}_{\mathrm{dG}}$ (depletion width at gate).Using abrupt junction approximation the $\mathrm{Y}_{\mathrm{dG}}$ (under dark condition) and $\mathrm{Y}^{\prime}{ }_{\mathrm{dG}}$ under illumination are calculated as given in [19]

$$
\begin{aligned}
Y_{d G}(x) & =\left(\frac{2 \varepsilon}{q N_{d r}}\left(V_{b i}+V(x)-V_{G S}\right)\right)^{1 / 2} \\
Y^{\prime}{ }_{d G}(x) & =\left(\frac{2 \varepsilon}{q N_{d r}}\left(V_{b i}+V(x)-V_{G S}-V_{o p}\right)\right)^{1 / 2}
\end{aligned}
$$

Where $\mathrm{V}(\mathrm{x})$ is channel voltage,

$\mathrm{V}_{\mathrm{bi}}$ built in potential,

Due to the photo voltage developed the effective bias across gate changes to $\left(\mathrm{V}_{\mathrm{GS}}+\mathrm{V}_{\mathrm{OP}}\right)$ from $\mathrm{V}_{\mathrm{GS}}$.

\section{SMALL SIGNAL MODEL}

For the above structure the small signal model is proposed based on [20] as shown in Figure 2.

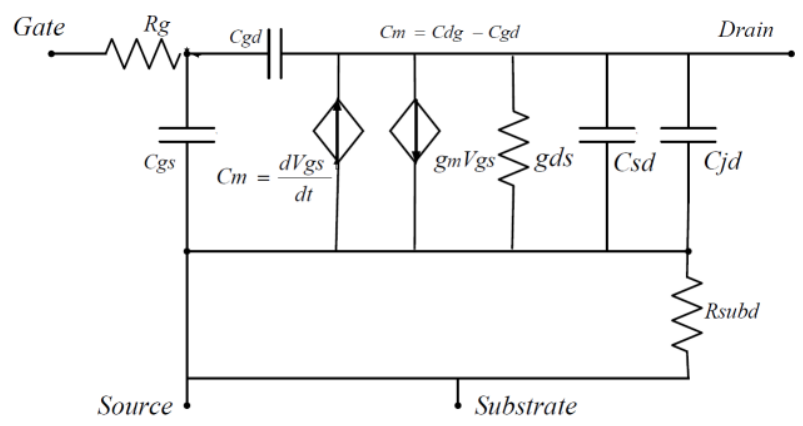

Figure 2 Equivalent circuit of MOSFET for Small signal analysis

For the present analysis, it is assumed that source and the substrate are shorted. The resistance $R g$ presents the effective gate resistance that represents channel resistance. $\mathrm{Cgs}, \mathrm{Cgd}$, $C d g$ and $C s d$, represent the capacitances in which intrinsic ,junction and overlap capacitances are merged. $\mathrm{Cm}(\mathrm{C} d g$ $\mathrm{Cgd}$ ) is the transcapcitance, $\mathrm{gm}$ is the transconductance and gds is the drain conductance. Compared to the model presented in [8-9], the drain to body (substrate) junction capacitance $\mathrm{Cjd}$ and drain to body (substrate) spreading resistance Rsubd are the additional elements in this model.

The Y parameters of a MOSFET in dark condition can be analytically evaluated from the following equations which are valid for frequency range upto $10 \mathrm{GHz}$ as per [19] and the circuit elements are modeled using[20] .

$$
\begin{aligned}
Y_{11}= & w^{2}\left(C_{g s}+C_{g d}\right)^{2} R_{g}+j w\left(C_{g s}+C_{g d}\right) \\
Y_{12}= & w^{2} C_{g d}\left(C_{g s}+C_{g d}\right) R_{g}-j w C_{g d} \\
Y_{12}= & g_{m}-w^{2} C_{d g}\left(C_{g s}+C_{g d}\right) R_{g}-j w C_{d g}-j w g_{m} R_{g}\left(C_{g s}+C_{g d}\right) \\
Y_{22}= & g_{d s}+\frac{w^{2} C_{j d} R_{s u b d}}{1+w^{2} C_{j d} R_{s u b d}}+w^{2} C_{d g} C_{g d} R_{g}+w^{2} g_{m} R_{g} C_{g d}\left(C_{g s}+C_{g d}\right) \\
& \frac{j w C_{j d}}{1+w^{2} C_{j d}{ }^{2} R_{s u b d}{ }^{2}}+j w C_{g d}+j w C_{s d} \\
& +j w g_{m} R_{g} C_{g d}-j w^{3} C_{g d} C_{d g}\left(C_{g s}+C_{g d}\right) R_{g}{ }^{2}
\end{aligned}
$$

Qtotal is charge calculated from equation (2). The transconductance and drain conductance of the device are calculated using equation (9a and $9 b$ ) where $\mathrm{I}_{\mathrm{DS}}$ is calculated as in equation (3). 


$$
\begin{gathered}
g_{m}=\frac{\partial I_{D S}}{\partial V_{G B}} \\
g_{d s}=\frac{\partial I_{D S}}{\partial V_{D B}}
\end{gathered}
$$

Bias dependence for capacitance and conductance is considered for calculation under dark and illuminated condition. The $\mathrm{S}$ parameters are obtained from $\mathrm{Y}$ parameters using $\mathrm{Y}$ to $\mathrm{S}$ conversion as follows [5].

$$
\begin{aligned}
& S_{11}=\frac{\left(\left(Y_{0}-Y_{11}\right)\left(Y_{0}+Y_{22}\right)+Y_{12} Y_{21}\right)}{\Delta Y} \\
& S_{12}=-\frac{2 Y_{12}}{\Delta Y} \\
& S_{21}=-\frac{2 Y_{21}}{\Delta Y} \\
& S_{22}=\frac{\left(\left(Y_{0}+Y_{11}\right)\left(Y_{0}-Y_{22}\right)+Y_{12} Y_{21}\right)}{\Delta Y} \\
& \Delta Y=Y_{11} Y_{22}-Y_{12} Y_{21}
\end{aligned}
$$

The expressions for gain and unilateral figure of merit are obtained from $\mathrm{S}$ parameters and reflection coefficients. The $\operatorname{source}\left(\Gamma_{\mathrm{S}}\right), \operatorname{load}\left(\Gamma_{\mathrm{L}}\right), \operatorname{input}\left(\Gamma_{\text {in }}\right)$ and $\operatorname{output}\left(\Gamma_{\text {out }}\right)$ reflection coefficients have same definitions as in [5]. The transducer power gain $G_{T}$, quantifies the gain of the amplifier placed between source and load.

$$
G_{T}=\frac{\left(1-\left|\Gamma_{L}\right|^{2}\right)\left|S_{21}\right|^{2}\left(1-\left|\Gamma_{S}\right|^{2}\right)}{\left|\left(1-S_{11} \Gamma_{S}\right)\left(1-S_{22} \Gamma_{L}\right)-S_{21} S_{12} \Gamma_{L} \Gamma S\right|^{2}}
$$

An often employed approximation for the transducer power gain is the so-called unilateral power gain, $\mathrm{G}_{\mathrm{TU}}$, which neglects the feedback effect of the amplifier $\left(\mathrm{S}_{12}=0\right)$. It is often used as a basis to develop approximate designs for an amplifier and its input and output matching networks.

$G_{T U}=\frac{\left(1-\left|\Gamma_{L}\right|^{2}\right)\left|S_{21}\right|^{2}\left(1-\left|\Gamma_{S}\right|^{2}\right)}{\left|1-\Gamma_{L} S_{22}\right|^{2}\left|1-S_{11} \Gamma S\right|^{2}}$

The available power gain for load side matching $\left(\mathrm{T}_{\mathrm{L}},=\right.$ Tout $)$ is defined as

$$
G_{A}=\frac{\left|S_{21}\right|^{2}\left(1-|\Gamma s|^{2}\right)}{\left(1-\left|\Gamma_{\text {out }}\right|^{2}\left|1-S_{11} \Gamma s\right|^{2}\right.}
$$

The operating Power Gain is the ratio of the power delivered to the load to the power supplied to the amplifier.

$$
G=\frac{\left(1-\left|T_{L}\right|^{2}\right)\left|S_{21}\right|^{2}}{\left(1-|\Gamma i n|^{2}\right)\left|1-S_{22} \Gamma_{L}\right|^{2}}
$$

Unilateral figure of merit is defined as

$$
U=\frac{\left|S_{12}\right|\left|S_{21}\right|\left|S_{22}\right|\left|S_{11}\right|}{\left(1-\left|S_{11}\right|^{2}\right)\left(1-\left|S_{22}\right|^{2}\right)}
$$

\section{RESULTS AND DISCUSSION}

Simulations has been carried out for an N-MOSFET device with $\mathrm{Nf}=10, \mathrm{Wf}=12 \mu \mathrm{m}$ and $\mathrm{Lf}=0.36 \mu \mathrm{m}$. Wf and $\mathrm{Lf}$ are effective width and length of single finger. $\mathrm{Nf}$ is the number of fingers. The parameters used for simulation are for $0.25 \mu \mathrm{m}$ CMOS process. Numerical calculations have been performed to evaluate the photo-voltage generated due to optical illumination. The ion implanted channel profile in the active region of the device is assumed to be non-uniformly doped with Gaussian profile. The resistances, capacitances and conductance are calculated considering bias dependence. The Y parameters of optically illuminated N-MOSFET, have been evaluated by numerical simulation in Matlab for quiescent condition of $\mathrm{V}_{\mathrm{GS}}=1 \mathrm{~V}, \mathrm{~V}_{\mathrm{DS}}=1 \mathrm{~V}$ and $\mathrm{V}_{\mathrm{GB}}=0$ for the frequency range varying from $100 \mathrm{MHz}$ to $10 \mathrm{GHz}$. S parameters are obtained from Y parameters. The characteristic impedance, load impedance and source impedance of the MOSFET is assumed to be $50 \mathrm{ohm}$ for gain analysis calculations.

The direct illumination of the gate results in generation of excess electron hole pairs due to absorption of radiation in depletion region. The excess carriers generated cause change in gate voltage due to photo voltage, $\mathrm{V}_{\mathrm{OP}}$ as reported in[13]

Parameters $\mathrm{Y}_{11}, \mathrm{Y}_{21}$ and $\mathrm{Y}_{12}$ in dark and illuminated condition remain the same as reported in[13], since there is no term in their expressions involving $\mathrm{R}_{\text {subd }}$ or $\mathrm{C}_{j d}$. This can be seen from the equations (8). Figure 3 is plot of real and imaginary parameter $\mathrm{Y}_{22}$ with and without substrate effect. It can be seen that real part of $Y_{22}$ significantly varies with substrate effect considerations, while the imaginary part remains the same. The effect due to substrate resistance and capacitance on imaginary part is almost negligible as the denominator term with $R_{\text {subd }}$ and $\mathrm{C}_{j d}$ elements in imaginary part evaluates to a very small value, and on other hand contributes in real $\mathrm{Y}_{22}$ at higher frequency due to $\mathrm{w}^{2}$ term.

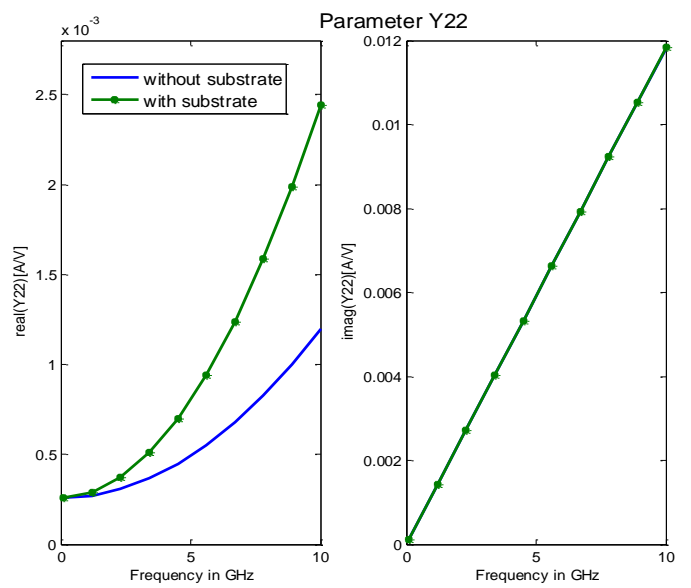

Figure 3 Real and Imaginary parameter y22 with and without substrate induced effect.

The $\mathrm{S}$ parameters are obtained from $\mathrm{Y}$ parameter as in equation (10a-10e) and all the expressions used for inter conversion involve parameter $\mathrm{Y}_{22}$. Due to this the substrate effect consideration affects all the $\mathrm{S}$ parameters. Figure 4 shows plot of S parameters with and without substrate effect in dark condition. It can be seen that seen that parameter $S_{21}$ and $\mathrm{S}_{12}$ are almost unaffected, and a change is seen in parameter $S_{11}$ and $S_{22}$ since $Y_{22}$ contributes in numerator and denominator inter-conversion expressions.

Figure 5 is Scattering parameter plot under dark condition and illuminated photon flux of $1 \times 10^{14}$ for quiescent condition of $\mathrm{V}_{\mathrm{GS}}=1 \mathrm{~V}, \mathrm{~V}_{\mathrm{DS}}=1 \mathrm{~V}$ and $\mathrm{V}_{\mathrm{GB}}=0$ for the frequency range varying from $100 \mathrm{MHz}$ to $10 \mathrm{GHz}$ with substrate induced effect. As expected and reported in [12], parameter 
$\mathrm{S}_{21}$ increases, which signifies forward gain of the device. This rise is due to increase in device transconductance in this region of device operation. To accommodate all $\mathrm{S}$ parameters on a single smith chart, the parameter $S_{21}$ and $S_{12}$ are downscaled and up -scaled by factor of 4 .

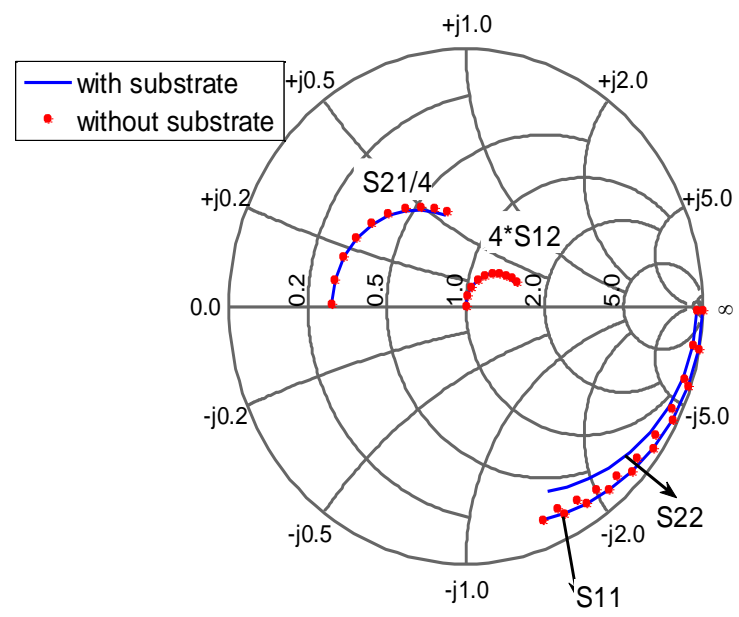

Figure 4. S Parameters with and without substrate induced effect in dark

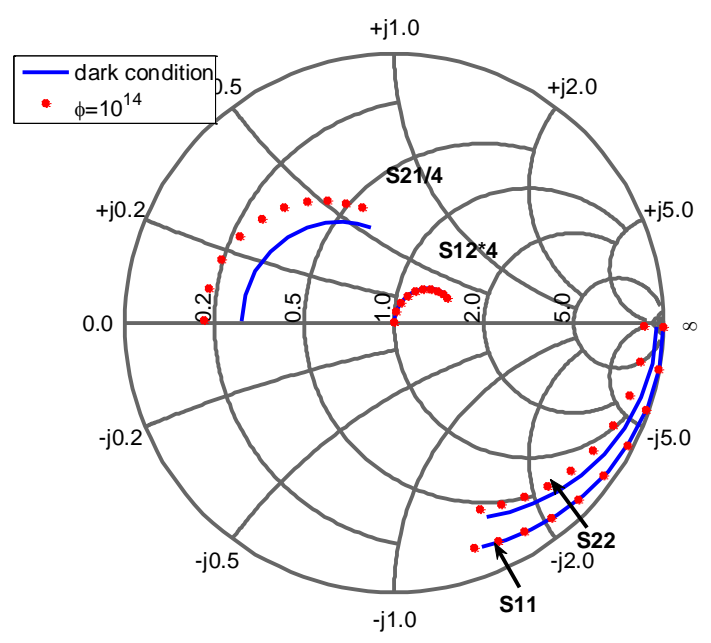

Figure 5 S Parameters in dark and illuminated condition

Figure 6 is plot of transducer gain, available gain and operating power gain under dark and illuminated condition of photon flux of $1 \times 10^{14}$ with respect to frequency. It can be seen that the transducer gain and operating power gain increase with optical radiation since the forward gain of the device enhances under optical radiation.

Figure 7 is plot of stable power gain, unilateral power gain and unilateral figure of merit. It can be seen that for both the cases gain improves with optical illumination and unilateral power gain almost matches transducer gain. Unilateral figure of merit accounts for error in neglecting the feedback gain i.e parameter $S_{12}$. Thus it should be as small as possible. This figure of merit is seen to reduce with photon flux impingement.

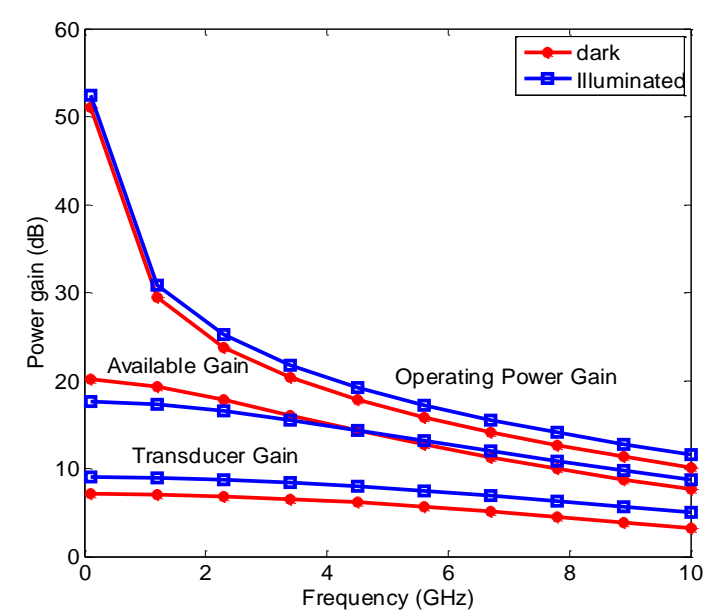

Figure 6. Transducer gain, available gain and operating power gain in dark and illuminated condition with photon flux of $1 \times 10^{14}$

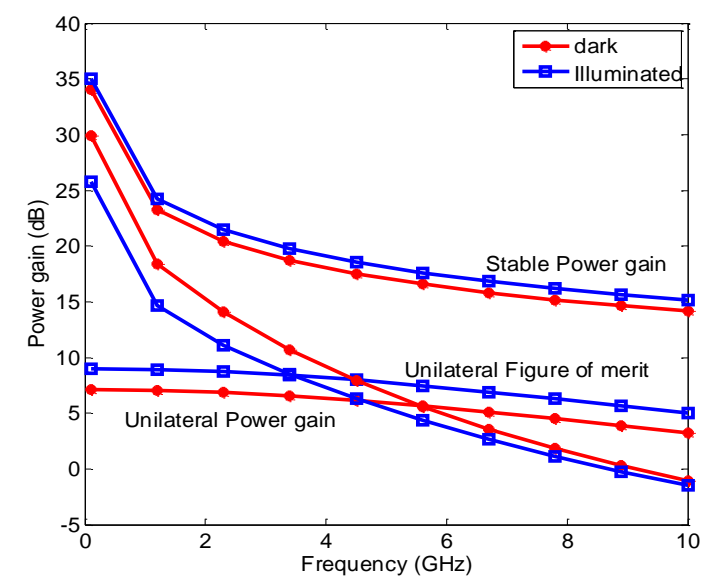

Figure 7. Unilateral gain, Maximum Stable Power gain and Unilateral figure of merit in dark and illuminated condition with photon flux of $1 \times 10^{14}$

Figure 8 shows variation of unilateral figure of merit with varying optical power at constant frequency of device operation of $5 \mathrm{GHz}$. It can be seen that this figure of merit decreases with increase of optical power indicating that worst case error will always reduce with optical illumination, since the parameter $S_{12}$ is almost constant with optical radiation while parameter $S_{21}$ enhances significantly.

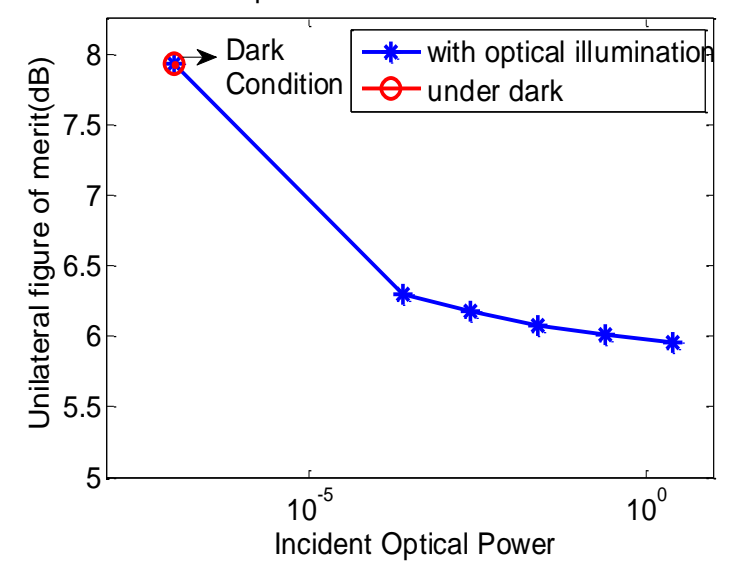

Figure 8 Unilateral figure of merit in dark and illuminated condition in dark and illuminated condition with photon flux of $1 \times 10^{14}$ to $1 \times 10^{18}$ 
Thus admittance parameter $Y_{21}$ and hence $S_{21}$ are enhanced with optical radiation due to increase in device current and transconductance. This is due to increase in optically generated carriers contributing to electron-hole pair generation. This also causes improvement in overall gain of interest and figure of merit of the device for the frequency range under consideration.

\section{CONCLUSIONS}

Theoretical investigations considering the substrate induced effects and analysis for various gain and unilateral figure of merit has been done on an MIS device, modified to optically gated MOSFET at microwave frequency. The increase in the photon flux density results in reduction in potential differences between the channel potential minimum and the source potential. The performance at RF indicates the device as promising candidate for optoelectronic applications like photo-detection and optical switching and imaging. Modification in device composition and materials is likely to make significant improvement in device figures of merits making it more useful in OEIC's.

\section{REFERENCES}

[1] Zimmermann,2000 Integrated Silicon Optoelectronics, Springer Series in Photonics.

[2] Anisha Goswami, et.al,,Substrate effect dependant scattering parameter extraction of short gatelength IGFET for microwave frequency applications, Microwave And Optical Technology Letters / Vol. 24, No. 5, March 52000

[3] Jasprit Singh,' Semiconductor Optoelectronics, Physics and Technology, McGraw-Hill, Inc.1995, ISBN 0-070S7b37-8

[4] M.Jamal Deen, Tor A. Fjeldly,2000, CMOS RF Modeling, Characterization And Applications, Selected Topics in Electronics and Systems - Vol. 24, World Scientific Publishing Co. Ltd.

[5] Reinhold Ludwig, 2000,"RF circuit Design”,LPE edition, Pearson education,2000,pp 456-495

[6] M. Berroth and R. Bosh,1990, Broad-band determinations of the FET small-signal equivalent circuits, IEEE Trans Microwave Theory Tech 38,pp. 891-895.

[7] P.J.V. Vandeloo,1989, Modelling of the MOS transistor for high frequency analog design, IEEE Trans ComputerAided Design, 713-722.

[8] C. Raynaud, J. Gautier, G. Guegan, M. Lerme, E. Playez, and G. Dambrine, High frequency performance of submicrometer channel-length silicon MOSFET's, IEEE Electron Device Letter 12(1991), 667-669.
[9] R. Sung, P. Bendix, and B.M. Das, Extraction of highfrequency equivalent circuit parameters of submicron gate length MOS-FETs, IEEE Trans Electron Devices 45 1998., 1769-1775.

[10] B.K.Mishra,P.N.Jain, G.Phade,2011,'Optically Controlled Transconductance Amplifier' ICWET' ACM 978-1-0449-8/11/02

[11] B.K.Mishra,G.Phade,P.Jain',2011,SmallSignal Modelling of illuminated MOSFET for RF Application' ICWET'ACM 978-1-4503-8/11/02.

[12] Jain P., Mishra B.K., Phade G.,2012, S Parameters of Optically Illuminated MOSFET, International Conference on Advancements in Engineering and Management(ICAEM)at Royal Institute of Technology And Science, Hyderabad.

[13] Christian C, EnzMOs transistor Modelling for RF IC Design, IEEE Transaction on solid state Circuits, Vol 35,Feb 2000,(186-201)

[14] Jain P., Mishra B.K., Phade G.,'AC Performance ofOptically Controlled MOSFET', Proceedings of SCEES, 2012,The IEEE website, doi : 10.1109 / SCEECS. 2012.618425, ISBN:978-1-4673-1516-6;

[15] B.K.Mishra,P.N.Jain,,S.C.Patil,,' Capacitance Modeling Of Optically Gated MOSFET', in proceedings of ACM, ICWET 2010, pp.887-891, February 2010.

[16] Dana Cristea, Silicon Opto-PET for Photonic Integrated Circuits, PROCEEDINGS EDMO 2001,IEEE,-78037049-

[17] B.K.Mishra, Lochan Jolly, S.C.Patil, " $\mathrm{In}_{1-\mathrm{x}} \mathrm{Ga}_{\mathrm{x}} \mathrm{As}$ a next generation material for photodetectors," Cyber journal, JSAM, pp.9-16, April 2011.

[18] Gayatri M Phade, B.K. Mishra, Prerana Jain,' Modeling of Optically Tailored Noise Parameters of MOSFET ', in IJCA Proceedings on International Conference in Computational Intelligence (ICCIA2012) iccia(8):-, March 2012

[19] B..K.Mishra (1995),Computer Aided modeling of solid state photodetectors, Ph.D thesis by, Birla institute of Technology, Mesra, Ranchi ,1995

[20] Minkyu Je, Ickjin Kwon, Hyungcheol Shin, And Kwyro Lee, Mosfet Modeling And Parameter Extraction For Rf IC's, International Journal of High Speed Electronics and Systems, Vol. 11, No. 4 (2001) 953-1006

[21] Y Cheng, MOSFET Modeling for RF IC Design, International Journal of High Speed Electronics and Systems, Vol. 11, No. 4 (2001) (1007-1084) 